\section{APPLICATIONS OF SPECTROSCOPY}

A CONFERENCE on the applications of spectroscopy to industrial and other problems was held, under the auspices of the London and Home Counties Branch of the Institute of Physies, at the Imperial College of Science and Technology on July 1 ; Dr. S. Whitehead presided.

The introductory address was given by Prof. H. Dingle, of the Imperial College, who opened with a short historical account of the development of spectroscopy. The pioneer work of Newton and, later, Fraunhofer, found no immediate practical application, and it was not until the middle of the nineteenth century that the subject began to acquire a general significance. Under the name of spectrum analysis, it was then regarded as a department of chemistry, and its discovery of new elements, coupled with the realization that a new and rapid method of chemical analysis had become possible, aroused hopes that a process of great practical value had been brought to light. These hopes were quenched, however, by the apparent capriciousness of the method, arising from eauses which, in the existing state of physical and chemical knowledge, it was impossible to understand, and chemists soon abandoned it almost entirely. For the next few decades spectroscopy was kept alive, and in some degree developed, by astronomers, for whom no other means of astrophysical investigation was available, and in spite of some unavoidable errors, their contribution was an essential preliminary to the great revival which eame with Bohr's theory of the hydrogen atom in 1913. For some dozen years thereafter, spectroseopy stood in the forefront of atomic physics, and the picture of the outer structure of atoms which eventually emerged was constructed pari passu with the explanation of the successive details of spectra.

This work, so far as atoms are concerned, is now almost complete, and one of its consequences is the rebirth of applied spectroscopy. Two main causes have been responsible for this. First, the requirements of physicists, in their enthusiasm to penetrate the secrets of atomic structure, impelled scientific instrument makers to turn their attention to the mass production of spectrographic apparatus, and this is now available in large quantity. Secondly, the newly acquired knowledge of the processes of production of spectra has made it possible to understand the vagaries which alienated the interest of earlier workers, and the spectrum has become a safe index to both the chemical composition and physical condition of the source of light. Prof. Dingle stated that the problem of qualitative spectrum analysis can now be regarded as solved; but considerable experience-not always possessed by the authors of published analyses-is necessary for trustworthy results. No general process of quantitative spectrum analysis, however, has so far been devised, although in certain special problems the method has been successfully used. He presented an analysis showing the various factors contributing to the strength of a photographed spectrum line, and described work which has been done at the Imperial College-and will be resumed as soon as conditions allow-directed towards the control of all of them except the amount of the corresponding element in the mixture to be analysed. The work of Mr. E. W. Foster, already published*, will, it is hoped, provide a basis for a process of quantitative spectrum analysis of great generality.

* Proc. Phys. Soc., 53, 594 (1941).
Mr. F. Twyman, managing director of Messrs. Adam Hilger, Ltd., followed with a description of certain spectroscopic instruments now available. He confined his remarks ehiefly to those which seem of most immediate importance--namely, instruments for analysis by emission spectra and those for identification and estimation of liquids or vapours by absorption in the visible, ultra-violet and infra-red regions. The number of spectrographic analyses of minerals and alloys in Great Britain alone must run into millions every year, while absorption spectra, particularly in the infra-red, have lately assumed great importance in problems of molecular structure of interest to the biochemist and the industrial chemist. The commonest instrument for the production of emission spectra is still the quartz spectrograph; but gratings, largely through the advocacy of G. R. Harrison in America, have recently come into more general use, and experience must decide which is to be preferred for practical purposes. In quantitative work an estimate of the strength of spectrum lines must be made, and for this purpose microphotometers, or micro-densitometers, are available. Attempts have been made to measure intensities directly by photo-electric cells, but there is little doubt that for a long time to come the spectrum will be recorded photographically and the strength of the lines measured by the blackening of the plate. Mr. Twyman next discussed improvements in sparking circuits, and concluded his remarks on instruments for emission spectra by a brief reference to the identification of chemical compounds by X-ray crystal analysis.

The most characteristic feature of apparatus for absorption spectra in the visible and ultra-violet regions is the photometric device, of which the first example was the sector spectro-photometer. The principle of this instrument is common to all subsequent photographic spectro-photometers, except very recent ones still in the experimental stage; but certain improvements in detail have been introduced, which are exemplified in the Spekker photo-electric absorptiometer. In the infra-red, where absorption spectra give important information bearing on the structure of organic molecules, the original Hilger instrument designed in 1909 is, with a few improvements, still in use to-day. Prisms of quartz, fluorite, rock-salt and sylvine are available, making investigation of the infra-red region possible up to at least $21 \mu$. The radiation is detected by a thermopile, and a recording form of the instrument has been devised.

The next speaker was Mr. D. M. Smith, of the Research Department of the British Non-Ferrous Metals Research Association. He discussed chiefly the difficulties met with in the spectrographic analysis of non-ferrous metals and alloys. In this work the chief problem is the quantitative determination of traces of impurities, and the methods adopted usually involve microphotometric measurements of are and spark spectrograms, especially the latter. Important sources of error lie in the lack of precise specification of sparking conditions, lack of complete understanding of the effect of small differences between the samples to be analysed and those used to provide standards for comparison, and uncertainties in plate calibration arising from imperfect design in certain types of microphotometer. The British Non-Ferrous Metals Research Association has for some time been collaborating with its members in attempting to improve the accuracy of spectrographic methods, four panels of the Research Sub-Committee having been formed. 
In addition to reports from the Photographic, Aluminium and Lead panels (the fourth panel deals with problems common to all the methods), further papers on specific applications are shortly to be published in book form under the title "Collected Papers on Metallurgical Analysis by the Spectrograph".

It is generally agreed that the standard alloys used for comparison should be closely similar in bulk composition and metallurgical history to the samples for analysis, and some pre-sparking is generally regarded as desirable in non-ferrous analysis, although in the analysis of steels it appears to be unnecessary. The 'direct comparison' method, due to A. Walsh, in which no internal standards are used, has proved successful in certain problems, but it is very susceptible to errors from incomplete control of the spark discharge. The reproducibility of spectrographic determinations is most concisely expressed by the standard deviation derived from an adequate number of repeat tests on the same sample, although the calibration of the plate by the use of standard alloys is a possible source of systematic error. In general, methods of compensation, based either on empirical relationships or on considerations of source temperature, are less satisfactory than improvements in the source of excitation as regards increasing analytical accuracy.

Dr. W. A. Roach, of the Elast Malling Research Station, then addressed the Conference on applications of spectroscopy to biological problems. Lundegardh (1928) and Ramage (1929), he said, were two of the earliest workers to use emission spectroscopy for studying the biological importance of minerals. Ramage burnt the dried and powdered material, rolled in a filter paper, in front of the slit of a spectrograph. Lundegardh sprayed a solution of the ash into an oxy-coal gas flame. The are method has been used by Webb and Fearon (1937) to make a survey of elements occurring in living tissues, and by Lewis, who found that serious trouble in young animals on 'teart' pastures in the west of England was caused by an excess of molybdenum. Mitchell used Goldschmidt's cathode layer method of soil analysis for studying a serious trouble in sheep called 'pining', which was traced to a deficiency of cobalt. These and absorptiometer methods are now in routine use in a number of biological laboratories.

Similar methods, as well as others, are being used at the East Malling Research Station for the diagnosis of mineral deficiencies and excesses in plants. The limitations of plant analysis when employed alone are illustrated by facts first discovered by Passy (1910) when seeking the cause of widespread chlorosis (absence of chlorophyll) in an orchard. The trouble was cured by injecting an iron salt into the chlorotic trees; yet the percentage content of iron was highest in chlorotic foliage, lower in certain green branches and trees found in the orchard, and lowest in the trees injected with iron salt. The iron in the ehlorotic leaves must have been in a form useless to the plant. When iron in a useful form was injected, the leaves grew in proportion to the injected iron, which 'diluted' the useless iron obtained naturally. This type of faulty mineral uptake is known as 'limeinduced chlorosis', since it is common on soils containing excess of calcium carbonate.

A system of diagnosis is being built up by studying the mineral composition of plants in relation to their performance, supplemented when necessary by testing how far performance is improved by injecting more of any selected element into the plant. Healthy plants tend to have a characteristic composition, and a deficiency of any essential element can be diagnosed by analysis alone. When calcium occurs in excess, however, the normal content of any of the trace elements (iron, manganese, zinc, boron, copper, etc.) may prove inadequate because it may be in a state useless to the plant. Supplementary methods, such as plant injection and a study of plant symptoms which are often characteristic of mineral deficiencies, must then be employed.

The final address was given by Dr. R. W. B. Pearse, of the Imperial College, who spoke on applications of the band spectra of molecules. These fall into two classes : first, those in which direct use is made of the spectrum, and secondly, those in which the spectrum is analysed in order to determine the structure of the corresponding molecule. In applications of the first class, a band system, and not an individual band, is to be taken as the molecular analogue of a single line in the spectrum of an atom. By means of such systems the scope of analysis can be extended in several ways. Thus, inaccessible sources of light, such as comets for example, sometimes show the presence of many of their constituents by bands and not lines. Secondly, certain elements which give no line spectra in the ordinary regions of observation when subjected to are or spark conditions readily show bands if suitable elements are present with which they can combine. Thus, fluorine and chlorine can often be detected by bands of $\mathrm{CaF}$ and $\mathrm{CuCl}$; nitrogen and oxyge $\mathrm{n}$ in the atmosphere of a copper are show a spectrum of NO ; and the spectrum of a hydrogen flame shows $S_{2}$ bands when sulphur is present. Thirdly, glow discharges in vacuum tubes often show bands which indicate the nature and purity of the gases present, and give a clue to the origin of unwanted residual gases, for example, leakage from the air, presence of moisture, degassing of walls, decomposition of grease, etc. Fourthly, absorption spectra have ena bled certain complex molecules to be identified, and indicated changes in the constitution of such molecules by corresponding changes in the spectrum. Tables of data are now available for the identification of molecular spectra, and their use could be greatly extended.

Applications of the second class require a much more detailed study of the spectrum. The spectra of diatomic molecules can be analysed into bands and branches, and energy-level diagrams constructed. Molecular constants representing the levels give the separation of the constituent atoms, the moment of inertia of the molecule, its frequency of vibration and electronic energy in various states, and the law of force between the atoms. If the observations are sufficiently extensive, the energies of dissociation for the various states can be obtained. This knowledge can be used, with the co-operation of the mathematician and the physical chemist, to throw light on many chemical problems. The determination of temperature and detection of isotopes are further possible products of the analysis of molecular spectra.

A short discussion was held at the conclusion of the meeting, at which it was proposed by Dr. J. Convey, and seconded by Dr. H. Lowery, that in view of the great importance which applied spectroscopy has acquired in various fields, the Board of the Institute of Physics should be asked to arrange for the formation of a group for the study of the subject. The proposal met with general agreement, and is to be submitted to the Board. 